\title{
The Effect of Paternal Addiction on Adolescent Suicide Attempts: A Qualitative Study
}

\author{
Mohammad Rafi Bazrafshan, ${ }^{1}$ Farkhondeh Sharif, ${ }^{2, *}$ Zahra Molazem, ${ }^{1}$ and Arash Mani ${ }^{3}$ \\ ${ }^{1}$ Department of Nursing, Faculty of Nursing and Midwifery, Student Research Committee, Shiraz University of Medical Sciences, Shiraz, IR Iran \\ ${ }^{2}$ Department of Mental Health \& Psychiatric Nursing, Community Based Psychiatric Care Research Center, Faculty of Nursing and Midwifery, Shiraz University of Medical \\ Sciences, Shiraz, IR Iran \\ ${ }^{3}$ Research Centre for Psychiatry and Behavioral Sciences, Shiraz University of Medical Sciences, Shiraz, IR Iran \\ "Corresponding author: Farkhondeh Sharif, Department of Mental Health \& Psychiatric Nursing, Community Based Psychiatric Care Research Center, Faculty of Nursing and \\ Midwifery, Shiraz University of Medical Sciences, Shiraz, IR Iran. Tel: +98-7116475250, Fax: +98-7116474252, E-mail: fsharif@sums.ac.ir
}

Received 2014 August 25; Revised 2015 June 01; Accepted 2015 June 07.

\begin{abstract}
Background: Research shows that parents have an immense influence over their children's behavior during adolescence. Adolescents who grow up with parents who are addicted to drugs and alcohol are more likely to turn to self-destructive behaviors such as suicide attempts. Because addiction in Iran is a primarily masculine phenomenon and in traditional Iranian culture, the father is responsible for family affairs and is recognized as the family provider, paternal addiction affects adolescent children. However, the impact of this type of parental addiction on adolescent suicide attempts has received little attention.

Objectives: This study was conducted to assess the impact of paternal addiction on adolescent suicide attempts.

Patients and Methods: This was a qualitative study that used a grounded theory approach. Thirteen participants, from two hospitals in Shiraz (southern Iran), were interviewed. We used purposeful sampling, followed by theoretical sampling. Data were collected from in-depth semi-structured interviews. Data analysis was done using Strauss and Corbin's (1998) approach, followed by the constant comparative method.

Results: The mean age of the adolescents was $15.75 \pm 1.83$, and all of them were single. Five main themes were extracted from the data: mental health problems, relationship problems, financial problems, family problems, and imitation of risky behaviors.

Conclusions: The findings suggest that in addition to the need to consider paternal addiction in health care plans, it is also necessary to pay attention to other family members, including the adolescents themselves. Health care providers, including nurses, can use the findings from this study for the purposes of prevention and education.
\end{abstract}

Keywords: Adolescent, Behavior, Addictive, Qualitative Research, Suicide, Attempted

\section{Background}

Any non-fatal and deliberate self-injury in which a person intentionally and without interference from others puts him/herself at risk of death is referred to as a suicide attempt (1). Worldwide, the number of suicide attempts resulting in death for adolescents is higher than for other age groups $(2,3)$. In some provinces in Iran, suicide rates among adolescents are on the rise (4). The results of a study conducted in 2013 on 773 children who were referred to hospitals affiliated with Shiraz University of Medical Sciences showed that the cause of referral for 298 cases was attempted suicide, $42.6 \%$ of whom were between the ages of 12 - 18 years. The same researchers conducted a similar study in 2005 , and the difference between the two indicates a rapid increase in the rate of suicide attempts for this age group in the city of Shiraz (close to two-and-a-half times)(5).
Parental substance abuse has harmful effects on children of all ages $(6,7)$. Adolescents who grow up with parents who are addicted to drugs and alcohol are more likely to turn to self-destructive behaviors such as suicide attempts $(7,8)$. Alonzo and colleagues (2014) studied the impact of parental addiction on children's risk of suicide attempts in the United States and, after adjusting for sociodemographic and clinical variables such as age, gender, race, educational level, family income, and histories of depression, concluded that parental addiction nearly doubled the likelihood that children would attempt suicide in their lifetimes (9). Similarly, Fine and colleagues' (2012) research in Pretoria (South Africa) on adolescents with a history of suicide attempts showed that $45 \%$ of these adolescents' caregivers had a history of alcohol abuse and 14\% had a history of drug abuse; thus, they reported that a history of alcohol and drug abuse among caregivers in an important predictor of adolescent suicide attempts (10). 
While many studies have been conducted on the effects of maternal substance abuse on children, few studies have been conducted on the effects of paternal addiction $(6,11)$. Addiction in Iran is a primarily masculine phenomenon, and in the traditional Iranian culture, the father is responsible for providing for the family and meeting its needs; furthermore, adolescents are known in the community according to the names of their fathers $(6,12)$. Therefore, understanding paternal addiction is necessary when seeking to prevent adolescent suicide attempts and rehabilitate adolescents who have attempted suicide.

\section{Objectives}

Most studies on the relationship between parental addiction and adolescent suicide attempts are simple descriptive studies; thus, extensive and in-depth qualitative research is required to understand this complex phenomenon. Hence, this study examined the impact of paternal addiction on adolescent suicide attempts and identified the risk factors.

\section{Patients and Methods}

This was a qualitative study that used a grounded theory approach. This approach can help researchers to investigate basic social and psychological processes within a social context $(13,14)$. In this type of research, sampling is continued until the new code related to the phenomenon under the study is not found $(14,15)$.

The key participants in the study were eight adolescents (three boys and five girls), aged 14 - 18 years, who had attempted suicide with medication and had been referred to two hospitals affiliated with Shiraz University of Medical Sciences. All of their fathers were addicted to drugs or alcohol. The emergency ward physicians confirmed that these adolescents had attempted suicide.

First, the interviewer (nurse) visited the adolescents in the ward and tried to elicit their trust so that they might participate in the study. After they agreed to participate, they were interviewed between 72 - 96 hours after being discharged. The times for the interviews were based on the patient's medical status, availability, and preference. Exclusion criteria included the participant's inability to share their experiences, whether due to lack of motivation, severe mental disease such as acute psychosis, or major depression.

Purposeful sampling was used because most people who have attempted suicide in the city of Shiraz are referred to these hospitals, and researchers try to provide maximum variation when sampling, including gender, culture, and socioeconomic status.
As data analysis progressed, some codes were applied to the interactions of adolescents with healthy parents, and the medical team continued theoretical sampling until the saturation level. Therefore, to elicit more comprehensive data collection during the theoretical sampling, one nurse, one psychologist, one psychiatrist, and two healthy adolescent mothers with no history of mental illness participated in the study. Theoretical sampling was continued until the new code related to the phenomenon under the study was not found.

At the beginning of each interview, the interviewer introduced himself to the participants and explained the purpose of the study and interviews. The interviews were semi-structured and started with a general question ("Please describe a day in your life."). Based on the participants' responses, the interviewer asked follow-up questions. Depending on the participants' roles, other questions were asked, such as "Can you talk about addiction of your spouse?"

At the end of each interview, the interviewer asked the participants to talk about any important things that had not been mentioned during the interview. The interview sessions were held in a quiet place, and the participants were assured of privacy. The interviews were conducted from September 2013 to June 2014, and their mean duration was 70 minutes. The data were collected and analyzed simultaneously.

To facilitate data analysis, each interview was digitally recorded and transcribed into MAXQDA 10 Software. For statistical analysis, Strauss and Corbin's (1998) approach, the constant comparative method, was used (14). For open coding, the data were broken down into parts and basic concepts were extracted. Similar codes were grouped during the continual comparison process and inserted into a category that was determined by the properties of each of its concepts. Then the categories were extended and connected to sub-categories, in a process called axial coding. At this stage, the researchers used selective coding to refine the codes and integrate theory.

The researchers used the four criteria proposed by Lincoln and Guba credibility, dependability, confirmability, and transferability to increase the accuracy of the findings (15). The credibility of the data was increased by the researchers' prolonged involvement in the process of collecting and analyzing data from two hospitals and writing memos. They also confirmed the accuracy of that data analysis with three experts in qualitative research and checked the primary codes with some participants. The maximum sampling variation increased the data dependability and confirmability. Finally, data transferability was increased by the researchers providing a clear path, with sufficient detail, to how they arrived at their findings. 
The ethics committee of Shiraz University of Medical Sciences approved the study (CT-92-6746). All the participants, and also the parents of adolescents under 15 years of age, signed the informed consent forms that were provided in accordance with the Helsinki Declaration.

\section{Results}

The mean age of the adolescents in this study was 15.75 \pm 1.83 , and all of them were single. Seven of their fathers were addicted to various drugs, and one was addicted to alcohol. Our findings showed that the addicted fathers had five problems: in mental health, relationships, finances, family breakdown, and risky behaviors, all of which can trigger adolescent suicide attempts.

\subsection{Mental Health Problems}

Paternal addiction could cause mental health problems for adolescents, which may eventually lead to suicide attempts. The most common adolescent mental health problems included depression, feelings of hopelessness, feelings of worthlessness, and shame. One participant said: "my father has an addiction to drugs he sleeps in bed until 10 - 11 in the morning. Sometimes, I came back from school by 2 pm and my dad was still sleeping. I had a lump in my throat, but I did not cry; I could not calm down. Why is my father's life like that? My dad's addiction has led to my depression. When I took the pills, I thought of my dad's addiction." One participant felt disappointed about the impact of addiction on his father and said: "Since high school, I have had suicidal thoughts. Life was very disappointing. Why is our life not like the lives of others? My father was sleeping all the time or was looking for a place to use drugs. I swear I have no hope in life suicide is better than having to live with such a human." One participant told about the feelings of worthlessness: "What is the worth of my life? We do not have a good home and we have a dad who is a drug addict." One participant felt ashamed in front of other people: "Every day, neighbor girls ask me, where is your dad? My father has been gone from home for a month and still has not returned looking for drugs. I was always ashamed in front of neighbors. I was tired of this kind of life." One member of the treatment team added: "one of the problems of these families is paternal addiction adolescents would get depressed when they compare their own dad with those of their friends. These problems can motivate adolescent to suicide."

\subsection{Relationship Problems}

Paternal addiction can cause disruption of the parent-child relationship, which may appear as a poor emotional relationship or as a conflict. One participant said: "my dad is a habitual drunkard. I have no relationship with my father for me, Dad is dead if I had a good father, I could talk about my problems with him and I probably would not have used the pills." One participant, who attempted suicide after a quarrel with his father, talked about the cause of the conflict: "My father is a drug addict recently; my father has not supported us. I said to him: what have you done for us? What type of father are you? When I quarreled with my dad, I wanted to kill myself."

\subsection{Financial Problems}

Many of the families also faced financial problems. Because the father is responsible for the family's needs, his addiction can affect the whole family, including the adolescents. One participant said: "our family's financial problems put much pressure on me. My dad is a drug addict and does not work. At home, we always have to worry about financial problems. Why do I not have money like the other girls in my school? Whenever they want, they can have what they want, but what about me?" Another participant said: "My dad did not support us, he was unemployed. My mom was forced to work outside the home to support our life this bothers me. I do not like this kind of life." One member of the treatment team added: "Some of these families face financial problem when they are discharged from the hospital we know the family man is addicted him does not have any income. We see many adolescents here who have problems because of these things."

\subsection{Family Problems}

Paternal addiction also causes problems between the parents, which in turn affects the adolescents. These problems could be seen as a conflict between the parents or the breakdown of the family structure. One of the participants said: "my dad becomes nervous when he is hungover and beats my mom. Why do my parents not live like the rest of the family? Why do I not have peace at home? I would have comfort if I died." One participant also said: "My husband is a habitual drunkard and does not support us we separated with agreement. Right when I separated from my husband, my daughter used some pills. She felt sad about our divorce." One member of the treatment team added: "One of the reasons the adolescents attempt suicide is family problems. The families of these adolescents have many issues. The father may be addicted, leading to the parents' separation from each other."

\subsection{Imitation of Risky Behaviors}

A family history of suicide attempts, especially paternal suicide attempts, can increase the risk of adolescent suicide attempts. One participant said: "I had debate with 
my husband about unemployment and drinking. At that moment, he went into the room and tried to hang himself. We broke the door and saved him my daughter saw this scene. Now my daughter used the pills; she says 'I learned from my dad suicide is in our nature."

\section{Discussion}

Research suggests that negativity in adolescents' lives, such as parental addiction to drugs and alcohol, increases the likelihood of mental health problems (16). Additionally, when adolescents experience psycho-emotional problems, they may think the only way to escape their painful states is to commit suicide $(16,17)$.

Research shows that addicted fathers have trouble establishing intimate relationships with their own children. Also, in comparison to families without addiction, conflicts between family members are higher in families with addiction problems. Conflict with parents causes psycho-emotional problems such as despair and aggression, and these feelings might stimulate adolescent suicide attempts. Therefore, unstable parent-child relationships in such families threaten adolescents' mental health and increase the chance of mental disorders, leading to an increased risk of suicide attempts $(6,16-20)$.

In Iranian families, fathers play an important economic role. For this reason, paternal addiction can disrupt the family income (6). The results of the research show that family poverty and economic problems are the most important factors contributing to adolescent suicide attempts. Economic problems can lead to family instability, increasing the likelihood of conflict and mental health problems for adolescents $(17,19)$. Paternal addiction is also the most important cause of divorce and conflict between parents $(21,22)$. The results of the research show that divorce and conflicts between the parents can influence adolescent mental health problems and, thus, suicide attempts. Previous research also shows that addicts frequently attempt suicide and that committing suicide is contagious $(23,24)$. Thus, a paternal history of suicide attempts may motivate suicide attempts by other family members, including adolescents.

Despite the fact that attempting suicide is a complex and multidimensional phenomenon, the results of this study, along with other studies that have examined the impact of maternal addiction, show that parental addiction can increase adolescent suicide attempts (25-27). Adolescents show a spectrum of psychological reactions to paternal addiction; with time, they may even come to believe that suicide provides the only escape from their painful psychological situations.
The present study described the factors that influence adolescent suicide attempts in families with paternal addiction. The adolescents in this study were largely aware of their fathers' drug/alcohol use and its impact on their family lives. Thus, the results highlight the need to support these families and the importance of long-term trusting relationships with professionals for both children and parents. Health care providers, including nurses, can provide this support.

Health care providers can assess for the five categories of problems in families and perform the necessary care interventions for adolescents in these families. In addition to taking action to remedy the addicted father and providing specialized support systems for such families, the most important strategies to prevent self-destructive behavior in adolescents are educating them on coping strategies and treating their mental health problems as early as possible.

The limitations of this study were that the participants were only those adolescents who had been referred to specific hospitals and that data collection was difficult because of the social stigma around suicide. Thus, researchers should be cautious about generalizing the results of this study.

\section{Acknowledgments}

The present article was extracted from the thesis written by Mohammad Rafi Bazrafshan and was financially supported by Shiraz University of Medical Sciences (Grant No. 92 6746). The researchers would like to thank all the participants who contributed to the study. The authors would also like to thank Dr. Nasrin Shokrpour at the center for development of clinical research of Namazi hospital for editorial assistance.

\section{Footnote}

Authors' Contribution: Mohammad Rafi Bazrafshan developed the study design, conducted the interviews and analysis, ensured the study's trustworthiness, and drafted the manuscript; Farkhondeh Sharif was the supervisor who participated in the study design, supervised the coding and data analysis process, and revised the manuscript. Zahra Molazem and Arash Mani were the research consultants who participated and advised during the study.

\section{References}

1. De Leo D, Burgis S, Bertolote JM, Kerkhof AJ, Bille-Brahe U. Definitions of suicidal behavior: lessons learned from the WHo/EURO multicentre Study. Crisis. 2006;27(1):4-15. doi: 10.1027/0227-5910.27.1.4 [PubMed: 16642910]. 
2. Pajoumand A, Talaie H, Mahdavinejad A, Birang S, Zarei M, Mehregan $\mathrm{FF}$, et al. Suicide epidemiology and characteristics among young Iranians at poison ward, Loghman-Hakim Hospital (1997-2007). Arch Iran Med. 2012;15(4):210-3. [PubMed: 22424037].

3. Greydanus DE, Shek D. Deliberate self-harm and suicide in adolescents. Keio J Med. 2009;58(3):144-51. [PubMed: 19826208].

4. Mohammadkhani P, Mohammadi MR, Delavar A, Khushabi KS, Rezaei Dogaheh E, Azadmehr H. predisposing and precipitating risk factors for suicide ideations and suicide attempts in young and adolescent girls. Med J Islamic Republic of Iran. 2006;20(3):123-9.

5. Haghighat M, Moravej H, Moatamedi M. Epidemiology of Pediatric Acute Poisoning in Southern Iran: A Hospital-Based Study. Bull Emerg Trauma. 2013;1(1):28-33. [PubMed: 27162818].

6. Garrusi S, Amirkafi A, Garrusi B. Experiences of drug dependent fathers in relation with their children: a qualitative study. Addict Health. 2011;3(1-2):29-38. [PubMed: 24494114].

7. Christoffersen MN, Soothill K. The long-term consequences of parental alcohol abuse: a cohort study of children in Denmark. $J$ Subst Abuse Treat. 2003;25(2):107-16. [PubMed: 14629993].

8. Arendt M, Sher L, Fjordback L, Brandholdt J, Munk-Jorgensen P. Parental alcoholism predicts suicidal behavior in adolescents and young adults with cannabis dependence. Int J Adolesc Med Health. 2007;19(1):67-77. [PubMed: 17458326].

9. Alonzo D, Thompson RG, Stohl M, Hasin D. The influence of parental divorce and alcohol abuse on adult offspring risk of lifetime suicide attempt in the United States. Am J Orthopsychiatry. 2014;84(3):316-20. doi: 10.1037/h0099804. [PubMed: 24827026].

10. Fine G, Alison HC, Westhuizen D, Krüger C. Predicting frequency of suicide attempts of adolescent outpatients at Weskoppies Hospital using clinical and demographic characteristics. South Afr J Psychol. 2012;18(1):22-6.

11. McMahon T], Rounsaville BJ. Substance abuse and fathering: adding poppa to the research agenda. Addiction. 2002;97(9):1109-15. [PubMed: 12199823].

12. Khajedaluee M, Assadi R, Dadgar Moghadam M. Health-Related Quality of Life of Young Addict Women in Mashhad, IR Iran. Int J High Risk Behav Addict. 2013;2(2):87-91. doi: 10.5812/ijhrba.10296. [PubMed: 24971281].

13. Strauss AL, Corbin JM. Basics of Qualitative Research. 2 ed. London: SAGE Publications; 1998.

14. Polit DF, Beck CT. Essential of nursing research, methods, appraisal, and utilization. 6 ed. Philadelphia: Lippincott Williams \& Wilkins; 2006.

15. Streubert HJ, Carpenter DR. Qualitative research in nursing: advanc- ing the humanistic imperative. 5 ed. Philadelphia: Wolters Kluwer Health / Lippincott Williams and Wilkins; 2011.

16. Bannink R, Broeren S, van de Looij-Jansen PM, Raat H. . Associations between parent-adolescent attachment relationship quality, negative life events and mental health. PLoS One. 2013;8(11)

17. Herrera A, Dahlblom K, Dahlgren L, Kullgren G. Pathways to suicidal behaviour among adolescent girls in Nicaragua. Soc Sci Med. 2006;62(4):805-14. doi: 10.1016/j.socscimed.2005.06.055. [PubMed: 16098648].

18. Diaz R, Gual A, Garcia M, Arnau J, Pascual F, Canuelo B, et al. Children of alcoholics in Spain: from risk to pathology. Results from the ALFIL program. Soc Psychiatry Psychiatr Epidemiol. 2008;43(1):1-10. doi: 10.1007/s00127-007-0264-2. [PubMed: 17932609].

19. keyvanara M, Haghighat M. Sociocultural contexts of attempting suicide among Iranian youth: a qualitative study. Eastern Mediterranean Health J. 2011;17(6)::17(6):529-35.

20. Mahato B, Ali A, Jahan M, Verma AN, Singh AR. Parent-child relationship in children of alcoholic and non-alcoholic parents. Ind Psychiatry J. 2009;18(1):32-5. doi: 10.4103/0972-6748.57855. [PubMed: 21234159].

21. Zandiyeh Z, Yousefi $\mathrm{H}$. Woman's experiences of applying for a divorce. Iran J Nurs Midwifery Res. 2014;19(2):168-72. [PubMed: 24834086].

22. Jewkes R. Intimate partner violence: causes and prevention. Lancet. 2002;359(9315):1423-9. doi: 10.1016/S0140-6736(02)08357-5. [PubMed: 11978358].

23. Roy A. Risk factors for attempting suicide in heroin addicts. Suicide Life Threat Behav. 2010;40(4):416-20. doi: 10.1521/suli.2010.40.4.416. [PubMed: 20822368].

24. Nordentoft M. Prevention of suicide and attempted suicide in Denmark. Epidemiological studies of suicide and intervention studies in selected risk groups. Dan Med Bull. 2007;54(4):306-69. [PubMed: 18208680].

25. Slesnick N, Feng X, Brakenhoff B, Brigham GS. Parenting under the influence: the effects of opioids, alcohol and cocaine on mother-child interaction. Addict Behav. 2014;39(5):897-900. doi: 10.1016/j.addbeh.2014.02.003. [PubMed: 24589871].

26. Lam WK, Cance JD, Eke AN, Fishbein DH, Hawkins SR, Williams JC. Children of African-American mothers who use crack cocaine: parenting influences on youth substance use. J Pediatr Psychol. 2007;32(8):87787. doi: 10.1093/jpepsy/jsm015. [PubMed: 17522115].

27. Kerwin ME. Collaboration between child welfare and substanceabuse fields: combined treatment programs for mothers. J Pediatr Psychol. 2005;30(7):581-97. doi: 10.1093/jpepsy/jsi045. [PubMed: 16166247]. 